\title{
A CELL-BASED SMOOTHED THREE-NODE PLATE FINITE ELEMENT WITH A BUBBLE NODE FOR STATIC ANALYSES OF BOTH THIN AND THICK PLATES
}

\author{
Chau Dinh Thanh ${ }^{1, *}$, Vo Ngoc Tuyen ${ }^{2}$, Nguyen Hoang Phuc ${ }^{1}$ \\ ${ }^{1}$ HCMC University of Technology and Education, Ho Chi Minh City, Vietnam \\ ${ }^{2}$ Thuan Viet Construction and Investment Company, Ho Chi Minh City, Vietnam \\ *E-mail: chdthanh@hcmute.edu.vn \\ Received October 24, 2016
}

\begin{abstract}
This paper develops the cell-based (CS) smoothed finite element method for a three-node plate finite element with a bubble node at the centroid of the element. Based on the first-order shear deformation theory, the in-plane strains are smoothed on three non-overlapped subdomains of the element to transform the numerical integration of the element stiffness matrix from the surfaces into the lines of the subdomains. The shearlocking phenomenon, which occurs when the plate's thickness becomes small, is removed by employing the mixed interpolation of tensorial components (MITC). The present element, namely CS-MITC3+, passes the patch test and behaves independently from the sequence of node numbers of the element. Numerical results given by the CS-MITC3+ elements are better than the MITC3+ elements. As compared to other smoothed three-node plate finite elements, the CS-MITC3+ is a good competitor.

Keywords: Shear locking, MITC3+, cell-based smoothed technique, CS-MITC3+ plate elements.
\end{abstract}

\section{INTRODUCTION}

Over past several decades, many plate finite elements have been developed to analyze plate structures based on the Kirchhoff-Love and Reissner-Mindlin theories. To apply for thin plates, the Kirchhoff-Love theory requires the $C^{1}$-continuous approximations of the displacement fields to satisfy the condition of neglecting the transverse shear strains. This $C^{1}$-continuous requirement makes difficulty to build finite element approximations. In contrast, the Reissner-Mindlin theory needs $C^{0}$-continuous displacement approximations because the theory considers the transverse shear strains. It means that the Reissner-Mindlin theory can easily use approximations of isoparametric plate finite elements. However, the Reissner-Mindlin plate elements encounter the difficulty of the shear-locking phenomenon that occurs when the plate thickness approaches to zero.

(C) 2017 Vietnam Academy of Science and Technology 
To overcome the shear-locking phenomenon, such techniques as the reduced and selective integration [1], the discrete Kirchhoff theory (DKT) [2], the assumed strains [3], the assumed natural strains (ANS) [4], the enhanced assumed strains (EAS) [5], the discrete shear gap (DSG) [6], or the mixed interpolation of tensorial components (MITC) [7] have been proposed. In which, the MITC technique is one of the most prominent approaches because of spatially isotropic behavior, no spurious zero energy mode and no shear locking. According to the MITC technique, the transverse shear strains are separately interpolated and connected to the displacement approximations at typing points that are designed to satisfy the spatially isotropic behavior. The MITC technique has been derived for 4-node (MITC4), 8-node (MITC8), 9-node (MITC9), 16-node (MITC16) quadrilateral and 3-node (MITC3), 6-node (MITC6), 7-node (MITC7), 9-node (MITC9) triangular plate/shell finite elements [8-12]. In recent years, the MITC technique have been developed for the 4-node quadrilateral and 3-node triangular shell elements with a bubble node, namely MITC4+ and MITC3+ respectively [13,14].

With effort, Liu et al. [15] have proposed the smoothed finite element methods (S-FEM), which average strain fields over domains defined within an element or over adjacent elements sharing the common edges or nodes, respectively called the cell-based (CS), the edge-based (ES) and the node-based (NS) finite element methods (FEM). These approaches of the S-FEM have been successfully applied to improve accuracy of 2-dimensional, 3-dimensional problems, plates and shells made of isotropic, composited or functional graded materials [16-30], just mentioned a few. Particularly, the CS-, ES-, and NSFEM have been developed for the 3-node Reissner-Mindlin plate elements employing the DSG technique to remove the shear locking, respectively called CS-DSG3, ES-DSG3 and NS-DSG3 elements [21-23]. The displacement fields of the DSG3 elements are approximated by the standard $C^{0}$-continuous shape functions. All the strain fields of the DSG3 elements, including the transverse shear strain modified by the DSG3 technique, are constant over each element but the values of these strains are dependent on the sequence of nodal numbering, meaning not spatially isotropic behavior. Therefore, when being separately averaged on three non-overlapped subdomains defined by element nodes and the centroid of the element, the strain fields of the CS-DSG3 elements are more accurate than the DSG3 elements [21].

In this paper, the CS-FEM is developed for 3-node Reissner-Mindlin plate finite elements that remove the shear-locking phenomenon by the MITC technique. Because of spatially isotropic behavior, the displacements given by the standard $C^{0}$-continuous plate elements with MITC3 technique [11] for the shear-locking elimination will be unchanged if the CS-FEM applied for the DSG3 are straightforwardly employed. By adding a bubble shape function for the approximations of the displacements, the strain fields of the 3node plate elements with a bubble node are not constant. Using the CS-FEM, the inplane strain fields are averaged on each subdomain of the element. The average is done by dividing integration of the in-plane strain fields over the subdomain's area. The area integration of the in-plane strains that contain derivatives of the shape functions are then transformed into the integration on the edges of the subdomains by the Green's theorem. As a result, element stiffness matrices are numerically integrated on lines instead of on surfaces and numerical errors caused by mesh distortion can be attenuated. To remove 
the shear-locking phenomenon, the MITC3+ technique [14] is employed to interpolate the transverse shear strains that connect the displacement approximations at typing points. The elements developed in this paper are called the CS-MITC3+ elements.

The paper is organized as follows. In the next section, the formulation of the CSMITC3+ elements is presented. In section 3, the robustness and accuracy of the displacements and bending moments given by the proposed elements are evaluated through static analyses of some plate problems. Conclusions are withdrawn in the last section.

\section{CS-MITC3+ PLATE FINITE ELEMENTS}

Consider a bending plate due to normal loads $p$ as shown in Fig. 1(a). Denote $h$ is the thickness and $\Omega \subset R^{2}$ is the area of the neutral plane. Assume the material is isotropic elastic and displacements of the plate are small. In the Cartesian coordinate system Oxyz, in which the plane $O x y$ is coincident with the neutral plane, the displacements of the first-order shear deformation plate are [31]

$$
u=z \beta_{x}(x, y), \quad v=z \beta_{y}(x, y), \quad w=w(x, y),
$$

in which, $u, v, w$ are, respectively, the translational displacements along $x-, y$ - and $z$ axis; $\beta_{x}, \beta_{y}$ are the rotations of vectors normal to the neutral plane about $y$ - and $x$-axis, respectively.

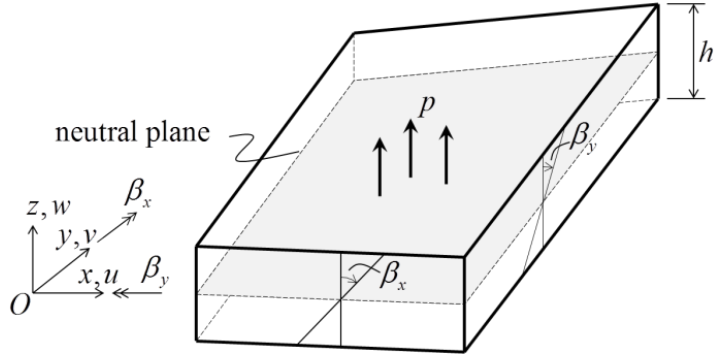

(a)

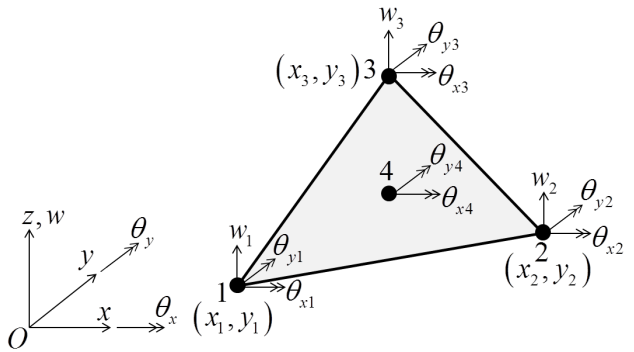

(b)

Fig. 1. (a) Mindlin-Reissner plate; (b) a 3-node triangular plate element with a bubble node

The plate is discretized by 3-node triangular finite elements $\Omega_{e}$ with a bubble node at the centroid of the element. The displacements of the Mindlin-Reissner plate elements are approximated as follows [14]

$$
w=\sum_{I=1}^{3} N_{I} w_{I}, \quad \beta_{x}=\sum_{I=1}^{4} N_{I} \theta_{y I}, \quad \beta_{y}=-\sum_{I=1}^{4} N_{I} \theta_{x I},
$$

where $w_{I}, \theta_{x I}, \theta_{y I}$ are the deflection and rotations about $x$ - and $y$-axis at node $I$ of an element with the positive directions defined in Fig. 1(b). $N_{I}$ are shape functions in the natural coordinate system $(\xi, \eta, \zeta)$ that include the cubic function $N_{4}$ corresponding to the bubble node at the centroid of the element

$$
N_{1}=1-\xi-\eta-\frac{1}{3} N_{4}, \quad N_{2}=\xi-\frac{1}{3} N_{4}, \quad N_{3}=\eta-\frac{1}{3} N_{4}, \quad N_{4}=27 \xi \eta(1-\xi-\eta)
$$


From Eqs. (1) and (2), the in-plane strains are

$$
\left\{\begin{array}{c}
\varepsilon_{x} \\
\varepsilon_{y} \\
\gamma_{x y}
\end{array}\right\}=z\left\{\begin{array}{c}
\beta_{x, x} \\
\beta_{y, y} \\
\beta_{x, y}+\beta_{y, x}
\end{array}\right\}=z\left\{\begin{array}{c}
\sum_{I=1}^{4} N_{I, x} \theta_{y I} \\
-\sum_{I=1}^{4} N_{I, y} \theta_{x I} \\
4 \\
\sum_{I=1}^{4} N_{I, y} \theta_{y I}-\sum_{I=1}^{4} N_{I, x} \theta_{x I}
\end{array}\right\}=z \mathbf{B}_{b} \mathbf{d}_{e} .
$$

Here $\mathbf{d}_{e}=\left[w_{1}, \theta_{x 1}, \theta_{y 1}, w_{2}, \theta_{x 2}, \theta_{y 2}, w_{3}, \theta_{x 3}, \theta_{y 3}, \theta_{x 4}, \theta_{y 4}\right]^{T}$ are the nodal displacements of an element and

$$
\mathbf{B}_{b}=\left[\begin{array}{ccccccccccc}
0 & 0 & N_{1, x} & 0 & 0 & N_{2, x} & 0 & 0 & N_{3, x} & 0 & N_{4, x} \\
0 & -N_{1, y} & 0 & 0 & -N_{2, y} & 0 & 0 & -N_{3, y} & 0 & -N_{4, y} & 0 \\
0 & -N_{1, x} & N_{1, y} & 0 & -N_{2, x} & N_{2, y} & 0 & -N_{3, x} & N_{3, y} & -N_{4, x} & N_{4, y}
\end{array}\right] .
$$

In the CS-MITC3+, the in-plane strains in each element are averaged over three subtriangular domains $\Omega_{e i}$ of areas $A_{e i}$ that are defined by connecting element nodes and the bubble node as demonstrated in Fig. 2. As a result, the in-plane smoothed strains are

$$
\left[\begin{array}{lll}
\tilde{\varepsilon}_{x} & \tilde{\varepsilon}_{y} & \tilde{\gamma}_{x y}
\end{array}\right]^{T}=z\left(\sum_{i=1}^{3} \frac{1}{A_{e i}} \int_{\Omega_{e i}} \mathbf{B}_{b} \mathrm{~d} \Omega\right) \mathbf{d}_{e}=z \underbrace{\left(\sum_{i=1}^{3} \frac{1}{A_{e i}} \tilde{\mathbf{B}}_{b i}\right)}_{\tilde{\mathbf{B}}_{b}} \mathbf{d}_{e}=z \tilde{\mathbf{B}}_{b} \mathbf{d}_{e} .
$$

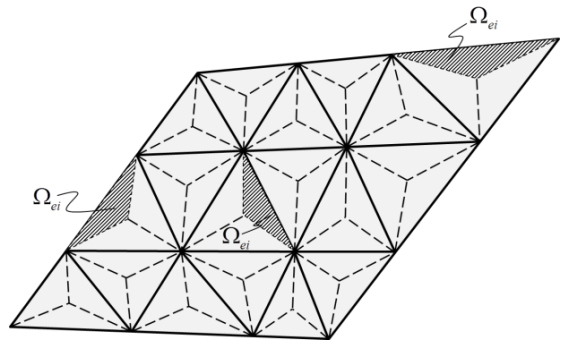

(a)

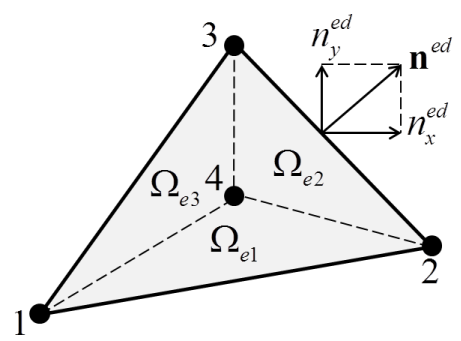

(b)

Fig. 2. (a) Mesh of the Mindlin-Reissner plate; (b) Definition of the subtriangular domains of a CS-MITC3+

Applying the Green's theorem, we have

$$
\begin{aligned}
& \tilde{\mathbf{B}}_{b i}=\int_{\Omega_{e i}} \mathbf{B}_{b} \mathrm{~d} \Omega
\end{aligned}
$$

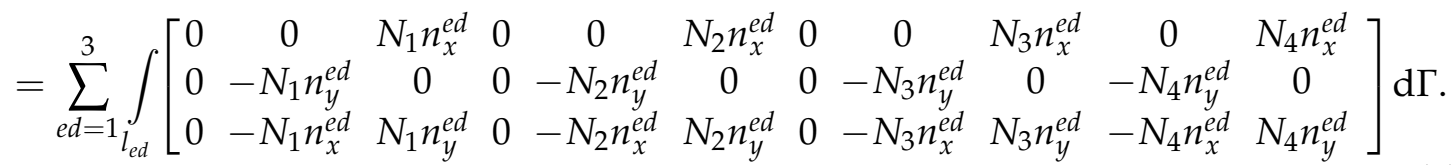


In which, $l_{e d}$ is the length of edge $e d$ of subtriangle $i ; n_{x}^{e d}$ and $n_{y}^{e d}$ are the direction cosines of the unit vector $\mathbf{n}^{e d}$ normal to edge $e d$ of subtriangle $i$ as illustrated in Fig. 2(b).

The covariant transverse shear strains are [7]

$$
\left\{\begin{array}{l}
\gamma_{\zeta \zeta} \\
\gamma_{\eta \zeta}
\end{array}\right\}=\left\{\begin{array}{l}
\mathbf{x}_{, \xi} \cdot \mathbf{u}_{, \zeta}+\mathbf{x}_{, \zeta} \cdot \mathbf{u}_{, \xi} \\
\mathbf{x}_{, \eta} \cdot \mathbf{u}_{, \zeta}+\mathbf{x}_{, \zeta} \cdot \mathbf{u}_{, \eta}
\end{array}\right\}
$$

Here, $\mathbf{x}, \mathbf{u}$ are respectively the coordinate and displacement vectors of a point in the plate.

If we use the displacement approximations given in Eq. (2) to compute the transverse shear strains in Eq. (8), the shear locking phenomenon will occur when the plate thickness decreases to zero. To overcome this phenomenon, we employ the assumed transverse shear strains designed for the continuum mechanics displacement-based shell finite elements MITC3+ [14]. According to this technique, the assumed covariant transverse shear strains are interpolated by

$$
\left\{\begin{array}{l}
\hat{\gamma}_{\xi \zeta} \\
\hat{\gamma}_{\eta \zeta}
\end{array}\right\}=\frac{2}{3}\left\{\begin{array}{l}
\gamma_{\xi \zeta}^{B} \\
\gamma_{\eta \zeta}^{A}
\end{array}\right\}-\frac{1}{3}\left\{\begin{array}{l}
\gamma_{\eta \zeta}^{B}-\gamma_{\xi \zeta}^{C}-\gamma_{\eta \zeta}^{C}-\hat{c}(3 \eta-1) \\
\gamma_{\xi \zeta}^{A}-\gamma_{\xi \zeta}^{C}-\gamma_{\eta \zeta}^{C}-\hat{c}(1-3 \xi)
\end{array}\right\},
$$

where $\hat{c}=\left(\gamma_{\xi \zeta}^{F}-\gamma_{\xi \zeta}^{D}\right)-\left(\gamma_{\eta \zeta}^{F}-\gamma_{\eta \zeta}^{E}\right)$ and $\gamma_{\xi \zeta}^{A}, \gamma_{\eta \zeta}^{A}, \gamma_{\xi \zeta}^{B}, \gamma_{\eta \zeta}^{B}, \gamma_{\xi \zeta}^{C}, \gamma_{\eta \zeta}^{C}, \gamma_{\xi \zeta}^{D}, \gamma_{\eta \zeta}^{E}, \gamma_{\xi \zeta}^{F}, \gamma_{\eta \zeta}^{F}$ are the covariant transverse shear strains at typing points $A, B, C, D, E$ and $F$ with the coordinates given in Fig. 3 . These covariant transverse shear strains are computed by substituting the displacement approximations in Eq. (2) into Eq. (8) and obtaining the values at the tying points. Therefore, expressing the assumed covariant transverse shear strains in terms of nodal displacements and transforming them into the global coordinates, we obtain

$$
\left[\begin{array}{cc}
\hat{\gamma}_{x z} & \hat{\gamma}_{y z}
\end{array}\right]^{T}=\hat{\mathbf{B}}_{s} \mathbf{d}_{e},
$$

in which, $\hat{\gamma}_{x z}, \hat{\gamma}_{y z}$ are the assumed transverse shear strains in the global coordinates, $\hat{\mathbf{B}}_{s}$ is the strain-displacement matrix.

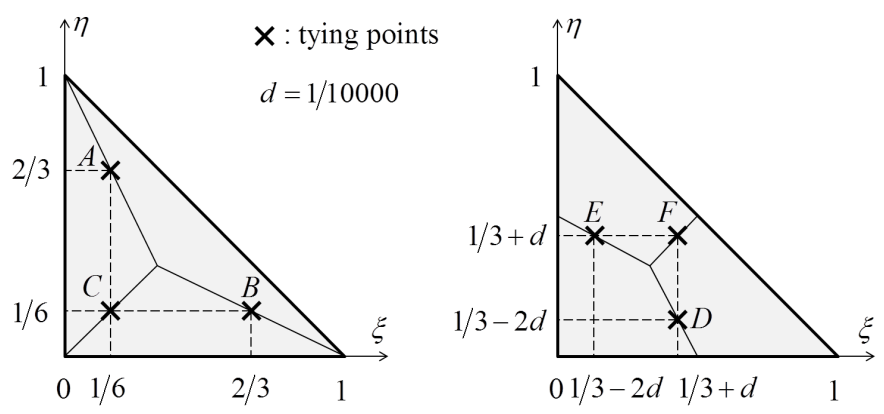

Fig. 3. Coordinates of typing points in the natural coordinates [14]

Using the smoothed in-plane strains in Eq. (6) and the assumed transverse shear strains in Eq. (10), the principle of virtual work for the CS-MITC3+ plate finite elements 
with the stabilized transverse shear strains [32] can be written

$$
\begin{gathered}
\int_{\Omega_{e}}\left(\int_{-h / 2}^{h / 2}\left(\delta \mathbf{d}_{e}^{T} \tilde{\mathbf{B}}_{b}^{T} z \mathbf{C}_{b} z \tilde{\mathbf{B}}_{b} \mathbf{d}_{e}\right) \mathrm{d} z\right) \mathrm{d} \Omega+\kappa \int_{\Omega_{e}}\left(\int_{-h / 2}^{h / 2}\left(\delta \mathbf{d}_{e}^{T} \hat{\mathbf{B}}_{s}^{T} \mathbf{C}_{s}^{*} \hat{\mathbf{B}}_{s} \mathbf{d}_{e}\right) \mathrm{d} z\right) \mathrm{d} \Omega=\int_{\Omega_{e}} \delta w p \mathrm{~d} \Omega \\
\Leftrightarrow \int_{\Omega_{e}} \delta \mathbf{d}_{e}^{T} \tilde{\mathbf{B}}_{b}^{T} \mathbf{D}_{b} \tilde{\mathbf{B}}_{b} \mathbf{d}_{e} \mathrm{~d} \Omega+\int_{\Omega_{e}} \delta \mathbf{d}_{e}^{T} \hat{\mathbf{B}}_{s}^{T} \mathbf{D}_{s}^{*} \hat{\mathbf{B}}_{s} \mathbf{d}_{e} \mathrm{~d} \Omega=\int_{\Omega_{e}} \delta \mathbf{d}_{e}^{T} \mathbf{N}^{T} p \mathrm{~d} \Omega \\
\Leftrightarrow \underbrace{\left(\tilde{\mathbf{k}}_{e}^{b}+\hat{\mathbf{k}}_{e}^{s}\right)}_{\mathbf{k}_{e}} \mathbf{d}_{e}=\mathbf{f}_{e} \Leftrightarrow \mathbf{k}_{e} \mathbf{d}_{e}=\mathbf{f}_{e} .
\end{gathered}
$$

Here, $\mathbf{k}_{e}=\tilde{\mathbf{k}}_{e}^{b}+\hat{\mathbf{k}}_{e}^{s}, \mathbf{k}_{e}^{b}=\int_{\Omega_{e}} \tilde{\mathbf{B}}_{b}^{T} \mathbf{D}_{b} \tilde{\mathbf{B}}_{b} \mathrm{~d} \Omega, \hat{\mathbf{k}}_{e}^{s}=\int_{\Omega_{e}} \hat{\mathbf{B}}_{s}^{T} \mathbf{D}_{s}^{*} \hat{\mathbf{B}}_{s} \mathrm{~d} \Omega, \mathbf{f}_{e}=\int_{\Omega_{e}} \mathbf{N}^{T} p \mathrm{~d} \Omega$ with

$\mathbf{D}_{b}=\int_{-h / 2}^{h / 2} z \mathbf{C}_{b} z \mathrm{~d} z=\underbrace{\frac{E}{1-v^{2}}\left[\begin{array}{ccc}1 & v & 0 \\ v & 1 & 0 \\ 0 & 0 & \frac{1-v}{2}\end{array}\right]}_{\mathbf{C}_{b}} \int_{h / 2}^{h / 2} z^{2} \mathrm{~d} z=\underbrace{\frac{E h^{3}}{12\left(1-v^{2}\right)}}_{D}\left[\begin{array}{ccc}1 & v & 0 \\ v & 1 & 0 \\ 0 & 0 & \frac{1-v}{2}\end{array}\right]$,

$\mathbf{D}_{s}^{*}=\int_{-h / 2}^{h / 2} \kappa \mathbf{C}_{s}^{*} \mathrm{~d} z=\kappa \underbrace{\frac{h^{2}}{h^{2}+\alpha h_{e}^{2}} \frac{E}{2(1+v)}\left[\begin{array}{ll}1 & 0 \\ 0 & 1\end{array}\right]}_{\mathbf{C}_{s}^{*}} \int_{-h / 2}^{h / 2} \mathrm{~d} z=\frac{\kappa h^{3}}{h^{2}+\alpha h_{e}^{2}} \frac{E}{2(1+v)}\left[\begin{array}{ll}1 & 0 \\ 0 & 1\end{array}\right]$,

$\mathbf{N}=\left[\begin{array}{lllllllllll}N_{1} & 0 & 0 & N_{2} & 0 & 0 & N_{3} & 0 & 0 & 0 & 0\end{array}\right]$,

and $E$ is the Young's modulus; $v$ is the Poisson's ratio; $D=E h^{3} /\left[12\left(1-v^{2}\right)\right] ; \kappa=5 / 6$ is the shear correction factor; $h_{e}$ is the longest length of the element sides; $\alpha$ is the stabilized parameter.

Static condensation is carried out on the element level for the rotational displacements of the bubble node. Therefore, the expression for Eq. (11) can be derived in terms of the nodal displacements at the vertices of the elements as follows

$$
\underbrace{\left[\begin{array}{cc}
\mathbf{k}_{e} & \mathbf{k}_{e b} \\
(9 \times 9) & (9 \times 2) \\
\mathbf{k}_{b e} & \mathbf{k}_{b} \\
(2 \times 9) & (2 \times 2)
\end{array}\right]}_{\mathbf{k}_{e}} \underbrace{\left\{\begin{array}{c}
\overline{\mathbf{d}}_{e} \\
(9 \times 1) \\
\mathbf{d}_{b} \\
(2 \times 1)
\end{array}\right\}}_{\mathbf{d}_{e}}=\underbrace{\left\{\begin{array}{c}
\overline{\mathbf{f}}_{e} \\
(9 \times 1) \\
\mathbf{0}(2 \times 1)
\end{array}\right\}}_{\mathbf{f}_{e}} \Leftrightarrow\left\{\begin{array} { l } 
{ \mathbf { k } _ { e } \overline { \mathbf { d } } _ { e } + \mathbf { k } _ { e b } \mathbf { d } _ { b } = \overline { \mathbf { f } } _ { e } } \\
{ \mathbf { k } _ { b e } \overline { \mathbf { d } } _ { e } + \mathbf { k } _ { b } \mathbf { d } _ { b } = \mathbf { 0 } }
\end{array} \Rightarrow \left\{\begin{array}{l}
\mathbf{k}_{e} \overline{\mathbf{d}}_{e}+\mathbf{k}_{e b}\left(-\mathbf{k}_{b}^{-1} \mathbf{k}_{b e} \overline{\mathbf{d}}_{e}\right)=\overline{\mathbf{f}}_{e} \\
\mathbf{d}_{b}=-\mathbf{k}_{b}^{-1} \mathbf{k}_{b e} \overline{\mathbf{d}}_{e}
\end{array}\right.\right.
$$

or

$$
\underbrace{\left(\mathbf{k}_{e}-\mathbf{k}_{e b} \mathbf{k}_{b}^{-1} \mathbf{k}_{b e}\right)}_{\overline{\mathbf{k}}_{e}} \overline{\mathbf{d}}_{e}=\overline{\mathbf{f}}_{e} \Leftrightarrow \overline{\mathbf{k}}_{e} \overline{\mathbf{d}}_{e}=\overline{\mathbf{f}}_{e} .
$$

Following the standard finite element procedure, we can obtain the equilibrium equation of the plate

$$
\mathbf{K d}=\mathbf{F} .
$$


In which, $\mathbf{K}$ and $\mathbf{F}$ are respectively the global stiffness and force matrices assembled from the matrices of element stiffness $\overline{\mathbf{k}}_{e}$ and force $\overline{\mathbf{f}}_{e} ; \mathbf{d}$ is the nodal displacements of the plate. Solving Eq. (13), we get the nodal displacements $\mathbf{d}$ and from $\mathbf{d}$ we can compute internal resultant forces of the plate.

\section{NUMERICAL RESULTS}

This section reports performance of the proposed elements upon basic tests and popular plate problems. The robustness and efficiency of the CS-MITC3+ elements are compared with other CS-DSG3 [21], ES-DSG3 [22], MITC3+ [14] and MITC4 [8] plate elements. Numerical investigations done by [32] shown that the stabilized parameter in range $0<\alpha<0.2$ reduces $L^{2}$-error of deflection, moment and shear force as compared to those given by the MITC4 elements with $\alpha=0$ for both simply supported and clamped plates. Here, the stabilized parameter $\alpha=0.1$ is chosen similarly to results provided by CS-DSG3 and ES-DSG3 [21]. Young's modulus $E=10.92$ and Poisson's ratio $v=0.3$ are used for the plate material in all numerical examples.

\subsection{Isotropic element test}

Consider a 0.01 thick plate discretized by one CS-MITC3+ element with three different nodal numbering sequences as demonstrated in Fig. 4. The plate is fixed at two nodes and loaded at the other node with $\mathbf{F}_{I}=\left[\begin{array}{lll}P & M_{x} & M_{y}\end{array}\right]^{T}=10^{-6}\left[\begin{array}{lll}1 & 2 & 0.5\end{array}\right]^{T}$. Numerical results in Tab. 1 show that the displacements are identical in the three cases of nodal numbering sequences. Therefore, the CS-MITC3+ elements pass the isotropic element test.

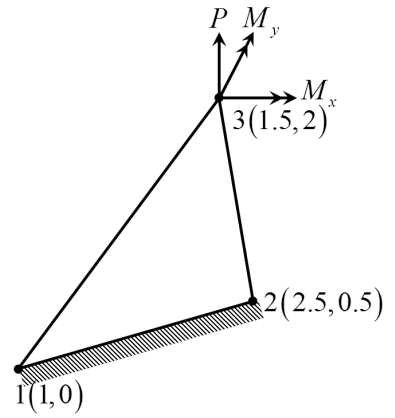

(a)

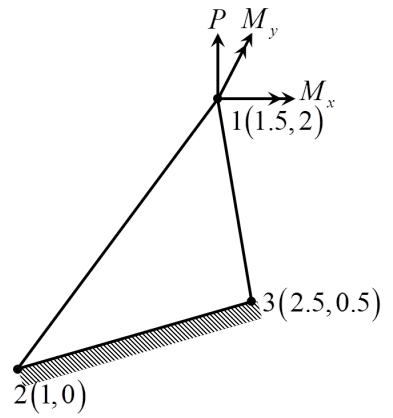

(b)

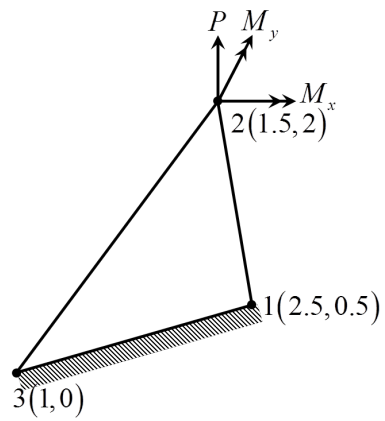

(c)

Fig. 4. Geometry, boundary, load and nodal numbering sequences for the isotropic element test

Table 1. Displacement results of the CS-MITC3+ isotropic element test

\begin{tabular}{|c|c|c|c|}
\hline Nodal numbering & $w$ & $\theta_{x}$ & $\theta_{y}$ \\
\hline Case (a) & 5.8519 & 6.2250 & 1.6751 \\
\hline Case (b) & 5.8519 & 6.2250 & 1.6751 \\
\hline Case (c) & 5.8519 & 6.2250 & 1.6751 \\
\hline
\end{tabular}




\subsection{Patch test}

A patch test with nodal coordinates and prescribed deflection $w=(1+x+2 y+$ $\left.x^{2}+x y+y^{2}\right) / 2$ [22] as shown in Fig. 5 is used to testify if the CS-MITC3+ elements are able to reproduce the displacements and internal forces. Assume the thickness is 0.01. Applying prescribed displacements computed from $w$ on the boundary nodes (node 1 to 4), the responses at node 5 given by CS-MITC3+ elements are listed in Tab. 2. Obviously, these responses are the same as the exact solutions, meaning that the CS-MITC3+ elements pass the patch test.

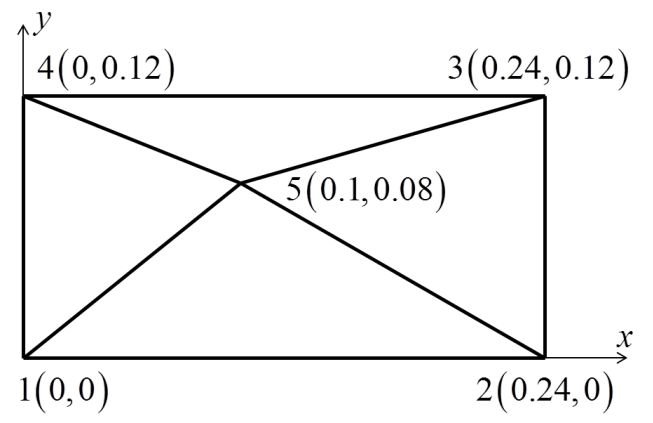

Fig. 5. Nodal coordinates of a mesh for the patch test

Table 2. Patch test results

\begin{tabular}{|c|c|c|c|c|c|c|}
\hline & $w_{5}$ & $\theta_{x 5}$ & $\theta_{y 5}$ & $M_{x 5} \times 10^{-5}$ & $M_{y 5} \times 10^{-5}$ & $M_{x y 5} \times 10^{-5}$ \\
\hline CS-MITC3+ & 0.6422 & 1.1300 & -0.6400 & -0.1300 & -0.1300 & -0.0350 \\
\hline Exact & 0.6422 & 1.1300 & -0.6400 & -0.1300 & -0.1300 & -0.0350 \\
\hline
\end{tabular}

\subsection{Simply supported square plate}

Consider a simply supported square plate subjected to uniform distributed loads $p=1$. Assume the length of the plate $L=1$ and $L / h=1000$ or 10 , equivalent to a thin or thick plate. Due to symmetry, a quarter of the plate is regularly discretized by $N_{x} \times N_{y}$ elements, in which $N_{x}=N_{y}=2,4,6,8,10,12$ are number of the elements on edges in $x$ and $y$-direction of the plate, respectively.

Fig. 6 presents the relation between the relative errors of the central deflection, which are given by the CS-MITC3+ and other elements, and the length of element size in the logarithm scale. As shown in Fig. 6, the convergence rates of these elements are similar but the accuracy provided by the CS-MITC3+ elements is better than the other three-node plate elements in cases of the thin and thick plates.

Nondimensional moments $M_{c} /\left(p L^{2} / 10\right)$, in which $M_{c}$ is the moment at the plate center, computed by the different elements against the different mesh densities $N_{x} \times N_{y}$ are listed in Tab. 3. The Tab. 3 also provides the relative errors of the central moments when the mesh $N_{x}=N_{y}=12$ is used. Compared with the CS-DGS3, ES-DSG3, MITC3+, the CS-MITC3+ gives better results for both thin and thick plates. However, the central 
deflection and moment of CS-MITC3+ are worse than the MITC4 elements, which use bilinear shape functions for the displacement approximations.

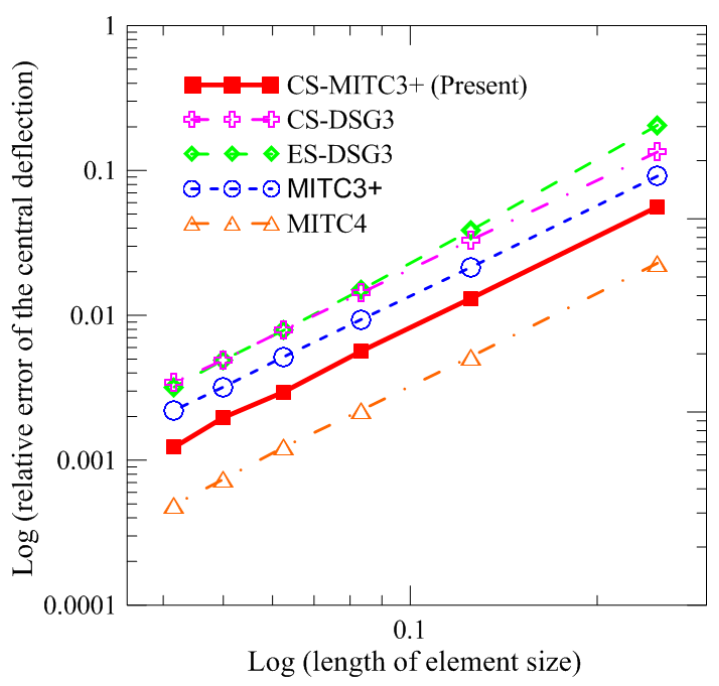

(a)

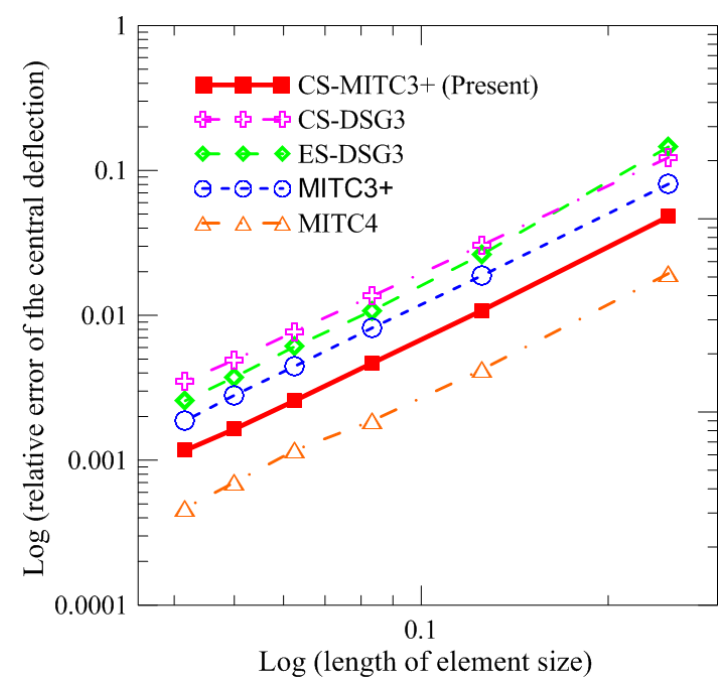

(b)

Fig. 6. Convergence rate of deflections at the plate center

Table 3. Nondimensional moment $M_{c} /\left(p L^{2} / 10\right)$ at the plate center

\begin{tabular}{|c|c|c|c|c|c|c|c|c|c|}
\hline \multirow{2}{*}{$L / h$} & \multirow{2}{*}{ Elements } & \multicolumn{6}{|c|}{$\operatorname{Mesh} N_{x} \times N_{y}$} & \multirow{2}{*}{$\begin{array}{l}\text { Relative } \\
\text { error (\%) }\end{array}$} & \multirow{2}{*}{$\begin{array}{l}\text { Analytical } \\
\text { results [33] }\end{array}$} \\
\hline & & $2 \times 2$ & $4 \times 4$ & $6 \times 6$ & $8 \times 8$ & $10 \times 10$ & $12 \times 12$ & & \\
\hline \multirow{5}{*}{1000} & CS-MITC3+ & 0.3827 & 0.4575 & 0.4701 & 0.4742 & 0.4760 & 0.4769 & 0.42 & \multirow{5}{*}{0.4789} \\
\hline & CS-DSG3 & 0.3721 & 0.4527 & 0.4673 & 0.4724 & 0.4747 & 0.4760 & 0.61 & \\
\hline & ES-DSG3 & 0.3687 & 0.4559 & 0.4692 & 0.4735 & 0.4755 & 0.4766 & 0.48 & \\
\hline & MITC3+ & 0.3822 & 0.4577 & 0.4702 & 0.4742 & 0.4760 & 0.4770 & 0.40 & \\
\hline & MITC4 & 0.4075 & 0.4612 & 0.4710 & 0.4745 & 0.4761 & 0.4769 & 0.42 & \\
\hline \multirow{5}{*}{10} & CS-MITC3+ & 0.3833 & 0.4573 & 0.4699 & 0.4741 & 0.4759 & 0.4769 & 0.42 & \multirow{5}{*}{0.4789} \\
\hline & CS-DSG3 & 0.3754 & 0.4533 & 0.4675 & 0.4725 & 0.4748 & 0.4760 & 0.61 & \\
\hline & ES-DSG3 & 0.3895 & 0.4591 & 0.4701 & 0.4740 & 0.4757 & 0.4767 & 0.46 & \\
\hline & MITC3+ & 0.3834 & 0.4574 & 0.4699 & 0.4740 & 0.4759 & 0.4769 & 0.42 & \\
\hline & MITC4 & 0.4075 & 0.4612 & 0.4710 & 0.4745 & 0.4761 & 0.4769 & 0.42 & \\
\hline
\end{tabular}

The limit where the present element can be free from the shear locking is investigated by computing the central deflection of the plate with the length-to-thickness ratio $L / h$ ranging from $10^{3}$ to $10^{8}$. As shown in Tab. 4, when using the mesh $N_{x}=N_{y}=12$, the CS-MITC3+ element and other smoothed plate elements like CS-DSG3, ES-DSG3 can give reasonable results until $L / h=10^{8}$ while the limits of $L / h$ for MITC3+ and MITC4 
are $10^{4}$ and $10^{5}$, respectively. It means that the proposed CS-MITC3+ can remove the shear locking very well.

Table 4. Nondimensional deflections at the plate center corresponding to $L / h$ from $10^{3}$ to $10^{8}$

\begin{tabular}{|c|c|c|c|c|c|c|c|}
\hline \multirow{2}{*}{ Elements } & \multicolumn{6}{|c|}{$L / h$} & \multirow{2}{*}{$\begin{array}{c}\text { Analytical } \\
\text { results [33] }\end{array}$} \\
\cline { 2 - 7 } & $10^{3}$ & $10^{4}$ & $10^{5}$ & $10^{6}$ & $10^{7}$ & $10^{8}$ & \\
\hline CS-MITC3+ & 0.4057 & 0.4057 & 0.4057 & 0.4057 & 0.4057 & 0.4057 & \\
\hline CS-DSG3 & 0.4048 & 0.4048 & 0.4048 & 0.4048 & 0.4048 & 0.4048 & \multirow{2}{*}{0.4062} \\
\hline ES-DSG3 & 0.4049 & 0.4049 & 0.4049 & 0.4049 & 0.4049 & 0.4049 & \\
\hline MITC3+ & 0.4053 & 0.4053 & 0.4047 & 0.3546 & 0.0342 & 0.0012 & \\
\hline MITC4 & 0.4060 & 0.4060 & 0.4060 & 0.4058 & 0.3941 & 0.2095 & \\
\hline
\end{tabular}

\subsection{Morley's skew plate}

Consider the simply supported Morley's skew plate of geometry and the ratio $L / h=100$ as shown in Fig. 7. The plate is subjected uniformed distributed loads $p=1$.

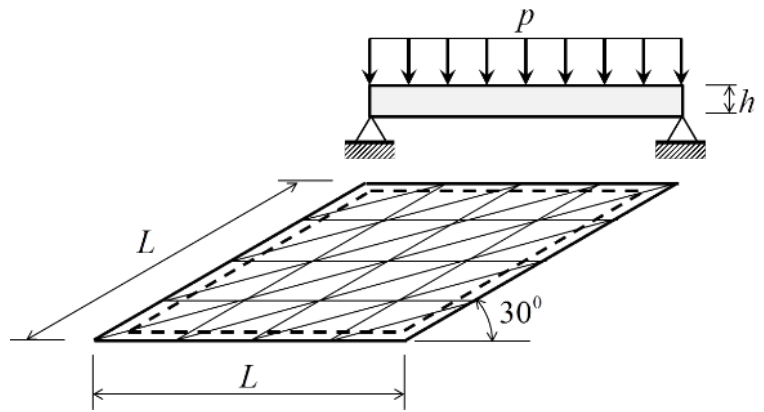

Fig. 7. Geometries, boundary conditions and $4 \times 4$ meshes of the Morley's skew plate

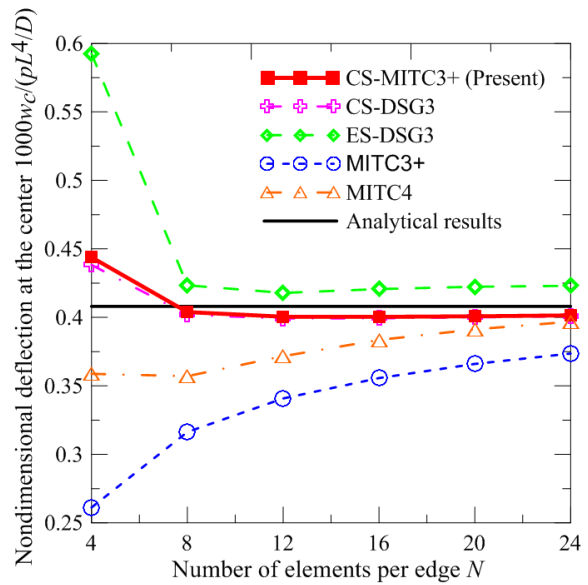

Fig. 8. Nondimensional deflections $1000 w_{c} /\left(p L^{4} / D\right)$ at the center of the Morley's plate

Regular meshes $N \times N$, in which $N=4,8,12,16,20,24$ are number of elements on each edge of the plate, are used to discretize the plate. Fig. 8 and Tab. 4 respectively show results of nondimensional deflection $1000 w_{c} /\left(p L^{4} / D\right)$ and moments at the plate center given by the CS-MITC3+ and the other elements using the different meshes. Compared with the original MITC3+ elements, the results provided by the CS-MITC3+ elements are greatly improved. The CS-MITC3+ elements also give results similar to other such strain smoothed elements as CS-DSG3 and ES-DSG3, and better than MITC4. 
Table 5. Normalized principal moments $100 M_{c} /\left(p L^{2}\right)$ at the center of the Morley's plates

\begin{tabular}{|c|c|c|c|c|c|c|c|c|}
\hline \multirow{2}{*}{ Elements } & \multicolumn{6}{|c|}{ Mesh $N \times N$} & \multirow{2}{*}{$\begin{array}{l}\text { Relative } \\
\text { error }(\%)\end{array}$} & \multirow{2}{*}{$\begin{array}{l}\text { Analytical } \\
\text { results [34] }\end{array}$} \\
\hline & $4 \times 4$ & $8 \times 8$ & $12 \times 12$ & $16 \times 16$ & $20 \times 20$ & $24 \times 24$ & & \\
\hline \multicolumn{9}{|c|}{ Normalized maximum principal moment } \\
\hline CS-MITC3+ & 1.4794 & 1.7712 & 1.8338 & 1.8570 & 1.8686 & 1.8758 & 1.79 & \multirow{5}{*}{1.9100} \\
\hline CS-DSG3 & 1.4773 & 1.7683 & 1.8313 & 1.8548 & 1.8668 & 1.8742 & 1.87 & \\
\hline ES-DSG3 & 1.6401 & 1.8333 & 1.8915 & 1.9237 & 1.8811 & 1.8884 & 1.13 & \\
\hline MITC3+ & 1.3125 & 1.5854 & 1.6834 & 1.7383 & 1.7742 & 1.7996 & 5.78 & \\
\hline MITC4 & 1.5097 & 1.6756 & 1.7608 & 1.8116 & 1.8425 & 1.8629 & 2.47 & \\
\hline \multicolumn{9}{|c|}{ Normalized minimum principal moment } \\
\hline CS-MITC3+ & 0.6199 & 0.8742 & 0.9685 & 1.0118 & 1.0341 & 1.0469 & 3.06 & \multirow{5}{*}{1.0800} \\
\hline CS-DSG3 & 0.6194 & 0.8729 & 0.9670 & 1.0103 & 1.0327 & 1.0456 & 3.19 & \\
\hline ES-DSG3 & 0.7384 & 0.9016 & 0.9952 & 1.0509 & 1.1398 & 1.1576 & 7.19 & \\
\hline MITC3+ & 0.5182 & 0.7441 & 0.8431 & 0.9004 & 0.9385 & 0.9659 & 10.56 & \\
\hline MITC4 & 0.6738 & 0.8590 & 0.9424 & 0.9923 & 1.0222 & 1.0421 & 3.51 & \\
\hline
\end{tabular}

In comparison with the lower bound solutions of the square and circular plates, see Tab. 3 and Tab. 6, the numerical results of the Morley's skew given by the CS-MITC3+ are upper bound. An intuitive explanation on the reason is that averaging strain fields over subdomains of the CS-MITC3+ elements overestimates the very high gradient of exact strain fields within elements at the acute angles of the skew plate.

\subsection{Circular plate}

Give a clamped circular plate of radius $R$ and thickness $h$ as illustrated in Fig. 9(a). Consider $R / h=50$ or 5 for the thin or thick plate respectively. The plate is applied uniform distributed loads $p=1$. Due to symmetry, we model and mesh one quarter of the plate by $N_{x} \times N_{y}$ elements, in which $N_{x}=N_{y}=2,4,6,8,10,12$ as shown in Fig. 9(b) for $N_{x}=N_{y}=4$.

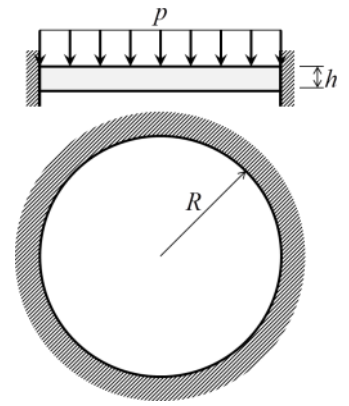

(a) Geometry, boundary and load of the circular plate

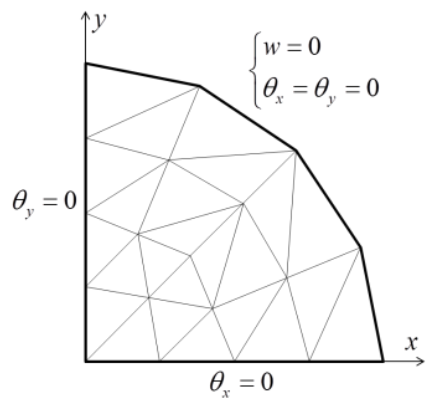

(b) Symmetric model and $N_{x} \times N_{y}=4 \times 4$ mesh

Fig. 9. Clamped circular plate subjected to a uniform distributed load and finite element model 
As shown in Fig. 10, for the thin and thick plates the central deflections given by the CS-MITC3+ are more accurate than those of CS-DSG3, ES-DSG3 and MITC3+ and similar to MITC4. However, Tab. 6 shows that the central moments in the finest mesh $\left(N_{x} \times N_{y}=12 \times 12\right)$ provided by CS-MITC3+ are the same as MITC3+, CS-DSG3 but not as good as the results given by MITC4, ES-DSG3 elements.

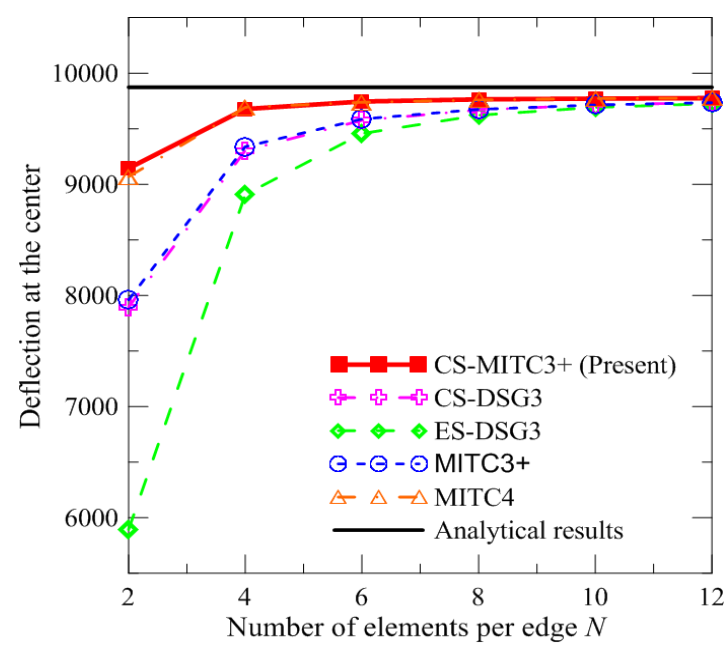

(a) $R / h=50$

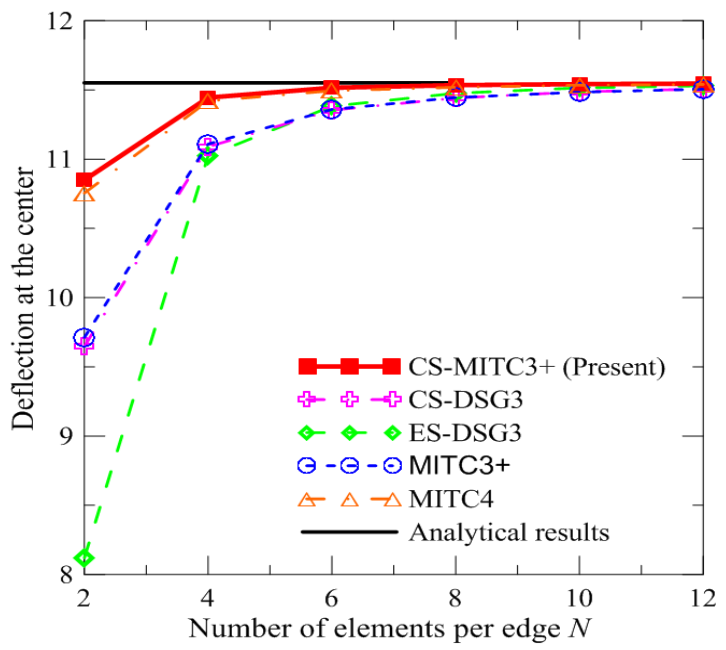

(b) $R / h=5$

Fig. 10. Deflections at the center of the clamped circular plate

Table 6. Moments at the center of the clamped circular plates

\begin{tabular}{|c|c|c|c|c|c|c|c|c|c|}
\hline \multirow{2}{*}{$R / h$} & \multirow{2}{*}{ Elements } & \multicolumn{6}{|c|}{$\operatorname{Mesh} N_{x} \times N_{y}$} & \multirow{2}{*}{\begin{tabular}{|l|} 
Relative \\
error $(\%)$
\end{tabular}} & \multirow{2}{*}{$\begin{array}{l}\text { Analytical } \\
\text { results [35] }\end{array}$} \\
\hline & & $2 \times 2$ & $4 \times 4$ & $6 \times 6$ & $8 \times 8$ & $10 \times 10$ & $12 \times 12$ & & \\
\hline \multirow{5}{*}{50} & CS-MITC3+ & 1.3621 & 1.8571 & 1.9556 & 1.9901 & 2.0061 & 2.0146 & 0.82 & \multirow{5}{*}{2.03125} \\
\hline & CS-DSG3 & 1.3326 & 1.8517 & 1.9559 & 1.9912 & 2.0059 & 2.0145 & 0.82 & \\
\hline & ES-DSG3 & 1.1736 & 1.8182 & 1.9523 & 1.9917 & 2.0061 & 2.0156 & 0.77 & \\
\hline & MITC3+ & 1.3597 & 1.8575 & 1.9555 & 1.9900 & 2.0060 & 2.0145 & 0.82 & \\
\hline & MITC4 & 1.8818 & 1.9404 & 2.0036 & 2.0127 & 2.0232 & 2.0255 & 0.28 & \\
\hline \multirow{5}{*}{5} & CS-MITC3+ & 1.3630 & 1.8538 & 1.9545 & 1.9899 & 2.0060 & 2.0146 & 0.82 & \multirow{5}{*}{2.03125} \\
\hline & CS-DSG3 & 1.3459 & 1.8517 & 1.9558 & 1.9913 & 2.0059 & 2.0145 & 0.82 & \\
\hline & ES-DSG3 & 1.2667 & 1.8513 & 1.9680 & 2.0003 & 2.0197 & 2.0256 & 0.28 & \\
\hline & MITC3+ & 1.3664 & 1.8549 & 1.9549 & 1.9900 & 2.0061 & 2.0146 & 0.82 & \\
\hline & MITC4 & 1.8350 & 1.9541 & 1.9949 & 2.0113 & 2.0232 & 2.0255 & 0.28 & \\
\hline
\end{tabular}




\section{CONCLUSIONS}

In this paper, the CS-FEM has been developed for three-node plate finite elements with a bubble node based on the first-order shear deformation theory. The in-plane strains are smoothed over non-overlapped subdomains of elements to transform the numerical integration of the element stiffness matrices from surfaces into lines. The transverse shear strains are interpolated by the MITC3+ technique to remove the shear-locking phenomenon.

The numerical tests shown that the CS-MITC3+ elements are free of the shear locking, spatially isotropic behaviour and pass the patch test. By employing the CS-smoothed technique, the CS-MITC3+ elements give more accurate displacements and bending moments than those given by the MITC3+ elements, especially in the skew Morley's plate. The numerical results of static analyses for both thin and thick plates also demonstrated that the CS-MITC3+ are good competitor as compared to such smoothed three-node plate elements as CS-DSG3 and ES-DSG3, which use the DSG technique for the shear-locking removal. Moreover, in spite of using only three-node, the CS-MITC3+ elements can give numerical results, especially in very thin plates, similar to or better than the four-node MITC4 elements. The present three-node plate elements with explicitly constant membrane and bending strains are useful for solving time-consuming problems with complicated geometry.

\section{REFERENCES}

[1] T. J. R. Hughes, M. Cohen, and M. Haroun. Reduced and selective integration techniques in the finite element analysis of plates. Nuclear Engineering and Design, 46, (1), (1978), pp. 203222. doi:10.1016/0029-5493(78)90184-x.

[2] J. L. Batoz, K. J. Bathe, and L. W. Ho. A study of three-node triangular plate bending elements. International Journal for Numerical Methods in Engineering, 15, (12), (1980), pp. 17711812. doi:10.1002/nme.1620151205.

[3] R. H. Macneal. Derivation of element stiffness matrices by assumed strain distributions. Nuclear Engineering and Design, 70, (1), (1982), pp. 3-12. doi:10.1016/0029-5493(82)90262-x.

[4] A. Tessler and T. J. R. Hughes. A three-node Mindlin plate element with improved transverse shear. Computer Methods in Applied Mechanics and Engineering, 50, (1), (1985), pp. 71-101. doi:10.1016/0045-7825(85)90114-8.

[5] U. Andelfinger and E. Ramm. EAS-elements for two-dimensional, three-dimensional, plate and shell structures and their equivalence to HR-elements. International Journal for Numerical Methods in Engineering, 36, (8), (1993), pp. 1311-1337. doi:10.1002/nme.1620360805.

[6] K. U. Bletzinger, M. Bischoff, and E. Ramm. A unified approach for shear-locking-free triangular and rectangular shell finite elements. Computers $\mathcal{E}$ Structures, 75, (3), (2000), pp. 321334. doi:10.1016/s0045-7949(99)00140-6.

[7] K. J. Bathe. Finite element procedures. Prentice Hall International, Inc, (1996).

[8] K. J. Bathe and E. N. Dvorkin. A four-node plate bending element based on Mindlin/Reissner plate theory and a mixed interpolation. International Journal for Numerical Methods in Engineering, 21, (2), (1985), pp. 367-383. doi:10.1002/nme.1620210213.

[9] K. J. Bathe and E. N. Dvorkin. A formulation of general shell elements-the use of mixed interpolation of tensorial components. International Journal for Numerical Methods in Engineering, 22, (3), (1986), pp. 697-722. doi:10.1002/nme.1620220312. 
[10] M. L. Bucalem and K. J. Bathe. Higher-order MITC general shell elements. International Journal for Numerical Methods in Engineering, 36, (21), (1993), pp. 3729-3754. doi:10.1002/nme.1620362109.

[11] P. S. Lee and K. J. Bathe. Development of MITC isotropic triangular shell finite elements. Computers \& Structures, 82, (11), (2004), pp. 945-962. doi:10.1016/j.compstruc.2004.02.004.

[12] K. J. Bathe, F. Brezzi, and S. W. Cho. The MITC7 and MITC9 plate bending elements. Computers $\mathcal{E}$ Structures, 32, (3-4), (1989), pp. 797-814. doi:10.1016/0045-7949(89)90365-9.

[13] Y. Ko, P. S. Lee, and K. J. Bathe. The MITC4+ shell element and its performance. Computers $\mathcal{E}$ Structures, 169, (2016), pp. 57-68. doi:10.1016/j.compstruc.2016.03.002.

[14] Y. Lee, P. S. Lee, and K. J. Bathe. The MITC3+ shell element and its performance. Computers E Structures, 138, (2014), pp. 12-23. doi:10.1016/j.compstruc.2014.02.005.

[15] G. R. Liu, K. Y. Dai, and T. T. Nguyen. A smoothed finite element method for mechanics problems. Computational Mechanics, 39, (6), (2007), pp. 859-877. doi:10.1007/s00466-006-00754.

[16] G. R. Liu and N. T. Trung. Smoothed finite element methods. CRC press, (2016). doi:10.1201/ebk1439820278.

[17] H. Nguyen-Xuan and G. R. Liu. An edge-based smoothed finite element method softened with a bubble function (bES-FEM) for solid mechanics problems. Computers $\mathcal{E}$ Structures, 128, (2013), pp. 14-30. doi:10.1016/j.compstruc.2013.05.009.

[18] G. R. Liu, T. Nguyen-Thoi, and K. Y. Lam. An edge-based smoothed finite element method (ES-FEM) for static, free and forced vibration analyses of solids. Journal of Sound and Vibration, 320, (4), (2009), pp. 1100-1130. doi:10.1016/j.jsv.2008.08.027.

[19] H. Nguyen-Xuan, T. Rabczuk, S. Bordas, and J. F. Debongnie. A smoothed finite element method for plate analysis. Computer Methods in Applied Mechanics and Engineering, 197, (13), (2008), pp. 1184-1203. doi:10.1016/j.cma.2007.10.008.

[20] T. Nguyen-Thoi, P. Phung-Van, H. Luong-Van, H. Nguyen-Van, and H. Nguyen-Xuan. A cellbased smoothed three-node Mindlin plate element (CS-MIN3) for static and free vibration analyses of plates. Computational Mechanics, 51, (1), (2013), pp. 65-81. doi:10.1007/s00466012-0705-y.

[21] T. Nguyen-Thoi, P. Phung-Van, H. Nguyen-Xuan, and C. Thai-Hoang. A cell-based smoothed discrete shear gap method using triangular elements for static and free vibration analyses of Reissner-Mindlin plates. International Journal for Numerical Methods in Engineering, 91, (7), (2012), pp. 705-741. doi:10.1002/nme.4289.

[22] H. Nguyen-Xuan, G. R. Liu, C. Thai-Hoang, and T. Nguyen-Thoi. An edge-based smoothed finite element method (ES-FEM) with stabilized discrete shear gap technique for analysis of Reissner-Mindlin plates. Computer Methods in Applied Mechanics and Engineering, 199, (9), (2010), pp. 471-489. doi:10.1016/j.cma.2009.09.001.

[23] H. Nguyen-Xuan, T. Rabczuk, N. Nguyen-Thanh, T. Nguyen-Thoi, and S. Bordas. A nodebased smoothed finite element method with stabilized discrete shear gap technique for analysis of Reissner-Mindlin plates. Computational Mechanics, 46, (5), (2010), pp. 679-701. doi:10.1007/s00466-010-0509-x.

[24] T. Nguyen-Thoi, P. Phung-Van, C. Thai-Hoang, and H. Nguyen-Xuan. A cell-based smoothed discrete shear gap method (CS-DSG3) using triangular elements for static and free vibration analyses of shell structures. International Journal of Mechanical Sciences, 74, (2013), pp. 32-45. doi:10.1016/j.ijmecsci.2013.04.005. 
[25] X. Cui, G. R. Liu, G. Y. Li, G. Y. Zhang, and G. Zheng. Analysis of plates and shells using an edge-based smoothed finite element method. Computational Mechanics, 45, (2), (2010), pp. 141-156. doi:10.1007/s00466-009-0429-9.

[26] S. Nguyen-Hoang, P. Phung-Van, S. Natarajan, and H. G. Kim. A combined scheme of edgebased and node-based smoothed finite element methods for Reissner-Mindlin flat shells. Engineering with Computers, 32, (2), (2016), pp. 267-284. doi:10.1007/s00366-015-0416-z.

[27] C. H. Thai, L. V. Tran, D. T. Tran, T. Nguyen-Thoi, and H. Nguyen-Xuan. Analysis of laminated composite plates using higher-order shear deformation plate theory and nodebased smoothed discrete shear gap method. Applied Mathematical Modelling, 36, (11), (2012), pp. 5657-5677. doi:10.1016/j.apm.2012.01.003.

[28] P. Phung-Van, T. Nguyen-Thoi, L. V. Tran, and H. Nguyen-Xuan. A cell-based smoothed discrete shear gap method (CS-DSG3) based on the C0-type higher-order shear deformation theory for static and free vibration analyses of functionally graded plates. Computational Materials Science, 79, (2013), pp. 857-872. doi:10.1016/j.commatsci.2013.06.010.

[29] L. Wu, P. Liu, C. Shi, Z. Zhang, T. Q. Bui, and D. Jiao. Edge-based smoothed extended finite element method for dynamic fracture analysis. Applied Mathematical Modelling, 40, (19), (2016), pp. 8564-8579. doi:10.1016/j.apm.2016.05.027.

[30] P. Liu, T. Q. Bui, C. Zhang, T. T. Yu, G. R. Liu, and M. V. Golub. The singular edgebased smoothed finite element method for stationary dynamic crack problems in 2D elastic solids. Computer Methods in Applied Mechanics and Engineering, 233, (2012), pp. 68-80. doi:10.1016/j.cma.2012.04.008.

[31] S. P. Timoshenko and S. Woinowsky-Krieger. Theory of plates and shells. McGraw-Hill, second edition, (1959).

[32] M. Lyly, R. Stenberg, and T. Vihinen. A stable bilinear element for the Reissner-Mindlin plate model. Computer Methods in Applied Mechanics and Engineering, 110, (3-4), (1993), pp. 343-357. doi:10.1016/0045-7825(93)90214-i.

[33] R. L. Taylor and F. Auricchio. Linked interpolation for Reissner-Mindlin plate elements: Part II-A simple triangle. International Journal for Numerical Methods in Engineering, 36, (18), (1993), pp. 3057-3066. doi:10.1002/nme.1620361803.

[34] L. S. D. Morley. Skew plates and structures. Pergamon Press, Oxford, (1963).

[35] R. Ayad, G. Dhatt, and J. L. Batoz. A new hybrid-mixed variational approach for ReissnerMindlin plates. The MiSP model. International Journal for Numerical Methods in Engineering, 42, (7), (1998), pp.1149-1179. doi:10.1002/(sici)1097-0207(19980815)42:7<1149::aidnme391>3.0.co;2-2. 